Projets

de paysage

\section{Projets de paysage}

Revue scientifique sur la conception et l'aménagement de l'espace

$22 \mid 2020$

Forêt et paysage

\title{
Les références spatiales et temporelles des paysages forestiers du rewilding en Europe : imaginaires, discours et projets
}

Spatial and Temporal References of the Rewilding of Forest Landscapes in Europe: Representations, Discourses and Projects

\section{Régis Barraud}

\section{OpenEdition}

\section{Journals}

Édition électronique

URL : http://journals.openedition.org/paysage/9076

DOI : $10.4000 /$ paysage.9076

ISSN : 1969-6124

Éditeur :

École nationale supérieure du paysage de Versailles-Marseille, Institut national des sciences appliquées Centre Val de Loire - École de la nature et du paysage, École nationale supérieure d'architecture et de paysage de Bordeaux, École nationale supérieure d'architecture et de paysage de Lille, Agrocampus Angers

Référence électronique

Régis Barraud, «Les références spatiales et temporelles des paysages forestiers du rewilding en Europe : imaginaires, discours et projets », Projets de paysage [En ligne], 22 | 2020, mis en ligne le 21 juillet 2020, consulté le 24 juillet 2020. URL : http://journals.openedition.org/paysage/9076 ; DOI : https://doi.org/10.4000/paysage.9076

Ce document a été généré automatiquement le 24 juillet 2020

Projets de paysage 


\title{
Les références spatiales et temporelles des paysages forestiers du rewilding en Europe : imaginaires, discours et projets
}

\author{
Spatial and Temporal References of the Rewilding of Forest Landscapes in \\ Europe: Representations, Discourses and Projects
}

Régis Barraud

1 À l'échelle européenne, depuis une vingtaine d'années, le rewilding est devenu l'un des processus polarisant les débats dans le champ de la conservation de la nature. Partant d'un courant hétérodoxe, d'abord développé en Amérique du Nord depuis l'approche militante et scientifique de la biologie de la conservation (Soulé et Noss, 1998), le rewilding alimente en Europe une controverse sociotechnique (Jørgensen, 2015; Lorimer et al., 2015) en même temps qu'il se traduit par des discours et des projets concrets (Jepson, 2016). Ceux-ci sont destinés à faire émerger de nouvelles formes de gestion et de culture de la nature. Il s'agirait, pour ses partisans, de faire davantage de place à une nature qui serait plus sauvage. Selon cette vision, l'ensauvagement serait l'un des remèdes pour pallier les effets des crises planétaires (changement climatique, effondrement de la biodiversité). Cela permettrait également d'atténuer les impacts psychosociaux d'une déconnexion des populations européennes à la nature. Cette (re)valorisation du sauvage s'accompagne d'une critique des modes de gestion patrimoniale des espaces naturels fondés sur la préservation de milieux et paysages issus d'héritages agropastoraux (Génot, 2014). De manière générale, alors que la gestion patrimoniale repose sur un pilotage de la biodiversité orienté par des interventions permanentes, les militants d'une Europe plus sauvage misent - à court ou moyen terme - sur la non-intervention et la libre évolution des milieux. Le rewilding est désormais porté par des ONG, des mécènes, des opérateurs écotouristiques et des scientifiques qui envisagent la valorisation de l'ensauvagement comme résultat de la déprise agricole à large échelle et/ou la mise en œuvre intentionnelle de projets de ré-ensauvagement. 
Ces deux volets, ensauvagement et ré-ensauvagement, peuvent servir de support à l'extension et/ou à la requalification de certaines aires protégées. Dans ce texte, nous désignerons l'ensemble des initiatives développées en ce sens à l'échelle européenne en tant que Mouvement de promotion de la Nature sauvage en Europe (MNSE). Nous avons proposé dans des publications précédentes (Barraud et Périgord, 2013; Barraud et al., 2019) un état des lieux et une première analyse des enjeux spatiaux et sociaux du déploiement de ce mouvement à l'échelle européenne. Le MNSE est constitué de courants et de clivages que nous serons amenés à présenter au fil du texte. Nous distinguerons dans le texte le rewilding CCC (pour cores, corridors, carnivores), tel que configuré initialement en Amérique du Nord, du rewilding herbivoriste, privilégiant les grands herbivores comme agents de ré-ensauvagement.

2 Cet article a pour objectif de prolonger le travail d'analyse du MNSE en centrant la réflexion sur les références spatiales et temporelles des paysages forestiers du rewilding. Plus précisément, il s'agira de comprendre comment ces références circulent, s'affrontent et évoluent au sein des imaginaires, des discours et des projets de réensauvagement. Les représentations savantes de la forêt se combinent avec ces images archétypales et contribuent à configurer des conceptions normatives du paysage (Debarbieux, 2005; Lepart et Marty, 2000). Les interprétations écologiques et paysagères de ce que pouvait être la forêt postglaciaire et de ce qu'elle pourrait ou devrait être sous l'effet du ré-ensauvagement traduisent une mise en tension des champs de l'expérience et des horizons d'attente (Claval, 2007).

3 Cet article est fondé sur la confrontation d'une revue de la littérature portant sur les natures boisées des paysages postglaciaires européens avec une approche documentaire multisource du mouvement de rewilding européen (presse, sites web, articles scientifiques). L'analyse du MNSE relève du décryptage de l'action d'ONG environnementales spécialisées dans la promotion du ré-ensauvagement de l'Europe et structurées en réseaux. Afin d'étudier la construction du discours et le contenu des projets de ces ONG, nous utilisons les sites Web associés en tant que sources et matériaux de recherche. Ce choix se justifie en raison de l'importance de la digitalisation des répertoires d'action et des modes d'expression des mobilisations environnementales (Ollitrault, 1999). Contrairement à ce qui pouvait être observé au début de ce processus dans les années 1990, le Web n'est plus seulement un support de communication parmi d'autres, il participe à la structuration des campagnes et à la diffusion des messages environnementaux. L'analyse des contenus Web des ONG est désormais une démarche reconnue dans le champ des humanités environnementales $(J$ ørgensen, 2017 ; Pezzullo et Cox, 2018). Dans le cadre de notre recherche, les sites web des ONG du MNSE ont été considérés en tant que complexe de production de discours composés de textes, d'images fixes, de vidéos, etc. Par ailleurs, les sites du MNSE médiatisent les actions concrètes de conservation engagées sur le terrain. Ces contenus numériques permettent donc de faire émerger une géographie du mouvement de rewilding en Europe. Les espaces qui fixent les projets de ré-ensauvagement et les projets eux-mêmes donnent lieu à des mises en récit élaborées (success stories). La mise au jour, dans les études de cas (partie 2), des références spatiales et temporelles des paysages forestiers est issue de l'analyse de ces récits ainsi que d'une mobilisation complémentaire de la littérature scientifique et de la presse qui documentent ces projets. 
4 Les études de cas (cf. figure 1) ont été retenues selon quatre critères principaux. Premièrement, les projets de rewilding portant sur la vallée de la Côa (Portugal) et sur les Highlands écossaises (la caledonian forest) sont représentatifs de la forte dynamique actuelle observée à l'Ouest de l'Europe, moins densément boisée, alors que le MNSE avait initialement investi plus fortement les forêts du Centre et de l'Est du continent où sont localisés les massifs les plus étendus et les mieux conservés à cette échelle. Deuxièmement, ces deux projets s'inscrivent dans deux réseaux différents qui portent des visions contrastées en matière de ré-ensauvagement. Troisièmement, ces deux terrains du rewilding sont très documentés ce qui permet de confronter plus efficacement les sources. Enfin, quatrièmement, ces deux exemples permettent de rendre compte de l'écart existant entre, d'une part, les conceptions climaciques anciennement ancrées dans la culture scientifique et populaire et, d'autre part, la grande diversité des paysages forestiers «manipulés» par les acteurs du réensauvagement.

5 La première partie de cet article a pour objectif, à partir d'une revue de la littérature académique et technique, de présenter de manière synthétique les conceptions relatives aux natures boisées des paysages postglaciaires européens. Il s'agit ici de montrer comment la vision d'une forêt originelle continue, dense et fermée est questionnée par d'autres interprétations scientifiques. Ces nouvelles lectures font émerger d'autres imaginaires et d'autres motifs paysagers. La deuxième partie a pour objectif de comprendre comment le mouvement contemporain du rewilding mobilise ces débats sur la forêt de référence. Ici, les acteurs de la conservation combinent des imaginaires de la nature et de nouveaux regards savants pour exprimer leurs conceptions de la forêt idéale. Nous verrons comment, dans cette perspective, l'agentivité des non-humains (Latour, 1999; Tsing, 2017), en particulier celle des carnivores et/ou des grands herbivores, est devenue un enjeu central dans le déploiement des stratégies de ré-ensauvagement. Cette partie permet d'appréhender deux échelles de productions de récits. La première relève de la stratégie de conservation à l'échelle européenne portée par les ONG et la seconde est celle des projets locaux de ré-ensauvagement. 
Figure 1. Forêts et mouvement de rewilding à l'échelle du continent européen

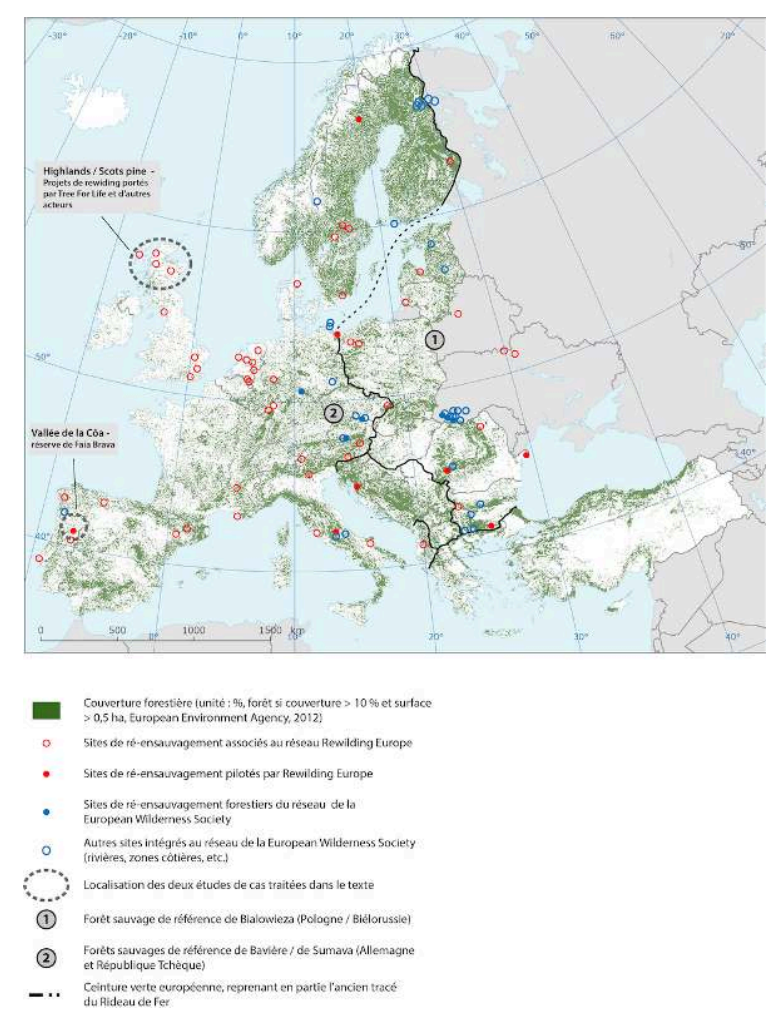

Sources : EEA, 2012/Rewilding Europe et European Wilderness Society Networks, 2020. Réalisation : Régis Barraud, 2020.

\section{Les natures boisées des paysages postglaciaires européens}

\section{La forêt primaire à canopée fermée}

6 Le premier modèle de référence en matière de conservation est constitué par celui de la forêt primaire alors que, partout dans le monde, ces écosystèmes très riches sont menacés. Le modèle de la forêt primaire et ses déclinaisons biogéographiques sont associés sur le plan épistémologique au développement des théories de successions végétales. Aboutissement de l'expression des processus climaciques, elles constituent un idéal type à partir duquel ont été pensés des états dégradés conduisant de manière régressive jusqu'aux formations les plus ouvertes. L'imagerie de la forêt primaire, malgré la diversité de ses expressions régionales et la dominance de ses versions tropicale et équatoriale, renvoie de manière assez nette à la canopée dense et luxuriante, composée de toutes les générations d'arbres, des plus anciens aux plus jeunes. La forêt primaire est souvent perçue et médiatisée à travers des traits caractéristiques : profusion du vivant et présence d'arbres morts, enchevêtrement du végétal hébergeant lui-même une faune très riche. Cette imagerie de la formation naturelle, parangon $\mathrm{du}$ sauvage, s'articule avec des imaginaires puissants ${ }^{1}$, historiquement ancrés et déclinés spatialement. La forêt primaire n'est pas seulement une référence scientifique fondamentale dans le développement de la biologie et de 
l'écologie scientifique, elle intègre le registre des cosmogonies forestières (Harrison, 1992).

7 Si Francis Hallé (cf. note infra 1) nous alerte sur l'urgence de la reconstruction de forêts primaires en Europe, c'est bien que celles-ci soient considérées comme étant présentes à l'état de fragments. Ces derniers témoigneraient, sous forme de peau de chagrin, d'une couverture ancienne beaucoup plus étendue et - en creux - ils traduiraient l'intensité de la dégradation anthropique de la forêt postglaciaire. Paradoxe de la transition forestière, la couverture boisée se développe en Europe depuis un " minimum » - situé dans la deuxième partie du xix ${ }^{e}$ siècle pour la France par exemple - mais la forêt considérée comme la plus authentique au regard du modèle climacique a largement disparu et les dernières reliques sont encore fragilisées par des entreprises extractivistes. Ainsi, dernièrement, la forêt de Bialowieza située à la frontière entre la Pologne et la Biélorussie a suscité de nouvelles inquiétudes.

Il n'est pas possible de revenir ici dans le détail sur la trajectoire historique et les caractéristiques de cette forêt-modèle. Les travaux de Simon Schama (1999) et ceux de Stuart Franklin (2002) permettront au lecteur qui le souhaite d'aller plus loin. Cependant, pour notre propos, il est important de retenir que l'idée selon laquelle Bialowieza serait une relique d'un manteau forestier européen étendu et quasi continu est ancienne (Hedemann, 1939). Plus largement, cette référence à un état forestier originel a été intégrée dans la rhétorique des spécialistes de la conservation et des écosystèmes forestiers. Ainsi, George Peterken, référence incontournable en matière d'écologie forestière, regrette, dans son ouvrage très diffusé Woodland Conservation and Management (1981, p. 3, première édition), la disparition de la forêt primaire qui, selon lui, couvrait à un moment donné une bonne partie du Royaume-Uni. En outre, le caractère sauvage actuel de la forêt de Bialowieza et la perception de sa précarité renforcent son inscription dans l'imaginaire des acteurs de la conservation de la nature (Carbonnelle et Lezaca-Rojas, 2017, p. 4-6).

9 Le récent article de Sabatini et al. (2017) illustre la réactivation de ce modèle de la forêt primaire dense, continue et d'origine postglaciaire en Europe. Les auteurs proposent un inventaire cartographique de ces fragments de forêt dont la couverture globale est estimée pour les zones documentées à 1,4 million d'hectares. Ces forêts sont bien représentées dans les Balkans et le Caucase et elles balisent le tracé de l'ancien rideau de fer. Ainsi, le registre de la forêt sauvage, incarnée par la mythique Bialowieza, est complété par d'autres "joyaux ». De manière pionnière, la libre évolution comme principe de conservation a été instituée pour les massifs transfrontaliers de la Bavière (Allemagne) et de Šumava (République tchèque), suscitant des réactions sociales d'abord contrastées puis de plus en plus positives (Barthod, 2010). Cette géographie des forêts sauvages européennes conduit Jean-Claude Génot (2010, p.17) à opposer un Ouest européen domestiqué à un Est européen sauvage. Ce sauvage de l'Est européen sert alors au naturaliste de terrain d'observation, de réflexion et de projection pour penser l'avenir de la nature en libre évolution à l'échelle continentale. Une telle spatialisation fournit des pistes concrètes pour le développement de stratégies de préservation et de rewilding. 


\section{Permanence et ajustements du modèle de la forêt primaire à canopée fermée}

Il convient de souligner que si le modèle de la forêt primaire dense et fermée demeure encore décisif dans la culture forestière comme dans celle de la conservation, les réflexions ont largement évolué dans la manière de considérer le caractère sauvage d'une forêt. La recherche compulsive des reliques de la couverture primaire a laissé place à une analyse plus ouverte sur le plan des temporalités et de la composition des états de référence. Ainsi, la diffusion des travaux nord-américains sur les forêts anciennes a conduit à un premier travail d'état des lieux à l'échelle européenne (Ribaut, 1991). Plus récemment, le couplage des notions de fonctionnalité, de naturalité et d'humanité (Vallauri, 2007) a contribué à fonder un programme de World Wildlife Fund (WWF) sur les forêts anciennes à haute valeur de conservation (2010-2013). Par ailleurs, la notion de féralitée étendue des animaux aux végétaux par Annik Schnitzler et JeanClaude Génot (2012), renouvelle encore plus profondément les codes d'identification et d'appréciation de la nature sauvage. Il ne s'agit plus seulement de préserver des reliques de forêt primaire mais d'assurer les conditions d'émergence et de développement pérenne de boisements en libre évolution. Francis Hallé propose de mobiliser cette notion de libre évolution afin de faire advenir la forêt primaire de demain en s'appuyant sur des noyaux de forêts naturelles. Ainsi, le modèle théorique et l'imaginaire de la forêt primaire sont toujours là, mais « la reconstruction » d'une telle forêt à large échelle (au moins 60000 hectares selon Hallé) passerait par la mise en œuvre d'un projet transgénérationnel sur sept à dix siècles, intégrant la question climatique planétaire (stockage du carbone).

\section{La thèse "Vera » : une forêt postglaciaire moins uniforme, plus ouverte et structurée par les grands herbivores}

11 La question de la physionomie de la forêt climacique en Europe a fait l'objet de discussions entre les écologues et les forestiers. Les divergences ont porté sur l'interprétation de la valeur écologique du climax, la composition en espèces et les processus mis en jeu. Le rôle joué par les grands herbivores dans ces trajectoires climaciques est interrogé dès les années 1980 comme le souligne Raphaël Larrère (1993). Cependant, ce sont bien les travaux de Frans Vera ${ }^{3}$ (2000) qui ont totalement bousculé le postulat de la "forêt à canopée fermée » en entraînant une série de polémiques. Frans Vera réexamine la période de référence durant laquelle se serait développée cette forêt (primaire) continue, à canopée fermée (-14 $000 \mathrm{BP}$ à $-5000 \mathrm{BP}$ ). Selon lui, le postulat initial doit être remis en cause par la réintégration dans l'analyse de données polliniques attestant de la présence généralisée dans les forêts tempérées de plaine du noisetier et du chêne qui ne peuvent se régénérer dans un contexte de canopée fermée. Par ailleurs, selon lui, l'hypothèse communément admise occulterait le rôle des grands herbivores dans la structuration et la dynamique évolutive de la forêt. De plus, la thèse de Vera remet en question l'idée selon laquelle toutes les formations boisées, ne correspondant pas au modèle de la canopée fermée, seraient le produit d'une dégradation anthropique que l'on pourrait positionner sur un gradient d'une succession régressive conduisant in fine à la prairie. 
12 Vera propose un modèle alternatif de cette forêt originelle et de son évolution. De manière ici simplifiée, nous pouvons reprendre les points saillants de sa théorie de cycle forestier complétée par Keith Kirby (2003). Ce cycle articule quatre séquences. La première est caractérisée par son ouverture forte (prairie, "parc»). La prairie comprend quelques patchs de broussailles et des arbres isolés. La deuxième phase permet le développement des broussailles. La troisième phase est celle du développement de bosquets dominant la broussaille par effet d'ombrage et abritant des grands ongulés. Les herbivores contribueraient alors à limiter la régénérescence des arbres et donc la fermeture du milieu, conduisant ainsi à la quatrième phase de réouverture du milieu (passage du boisement à la prairie).

13 La trajectoire des idées proposées par Vera est tout à fait remarquable : la controverse s'est développée dans de multiples directions. Cette controverse n'est pas restée cantonnée à une discussion théorique, elle a très rapidement été alimentée par la mise en œuvre opérationnelle des idées développées par Vera. Cela s'explique par le fait que Vera a forgé sa réflexion à partir de la bibliographie mais aussi à partir d'observations de terrain réalisées dans le cadre du projet de restauration écologique du polder d'Oostvaardersplassen (Vera, 2009). Ainsi, la discussion scientifique s'est fixée sur la question du degré d'ouverture de la forêt postglaciaire, sur le rôle des grands herbivores, sur la définition même de l'état de référence - considéré dans sa variabilité temporelle et spatiale - et également sur la valeur écologique globale des deux modèles discutés. On trouve peu de références aux publications et idées de Vera dans la littérature française. À titre d'exemple, dans la Revue forestière française, seul l'article de Xavier Rochel, publié en 2015, y fait explicitement référence et souligne leur importance. Les travaux de Vera, indépendamment des polémiques associées, ont conduit au réexamen d'objets spécifiques tels que les pré-bois ou forêts pâturées. Les géographes et les historiens spécialisés en géohistoire des paysages ruraux ont particulièrement investi ce champ (Hartel et Plieninger, 2014 ; Agnoletti et Emanueli, 2016). Leurs recherches ont conduit à étayer l'hypothèse d'une certaine continuité historique entre les paysages forestiers postglaciaires et certains paysages boisés issus des agricultures élaborées à partir du Néolithique. Dans cette perspective, les paysages semi-ouverts du début de l'Holocène auraient été - au moins partiellement - repris et ajustés par les pratiques agro-sylvo-pastorales (Rotherham, 2014). Ces auteurs ruralistes tendent à rejeter le rewilding passif et, au contraire, ils plaident pour la défense et la patrimonialisation des paysages boisés semi-ouverts façonnés par les hommes. Cette analyse déplace dans le temps le rôle décisif des sociétés européennes dans le processus de dégradation des environnements boisés : la rupture écologique et culturelle se produit à partir de la première industrialisation et se prolonge à travers un processus d'abandon des terres agricoles marginales (Rotherham, 2013). Cette interprétation ne semble toutefois pas totalement être en accord avec le discours de Vera lui-même. En effet, ce dernier regrette dès la préface de son ouvrage (2000, p.15-16) avoir dû affronter, lors de ses expérimentations à Oostvaardersplassen, l'opposition de certains environnementalistes attachés à la production d'un paysage géré sur la base de pratiques agricoles. De plus, il récuse le recours à la référence agropastorale comme base de la conservation en raison des effets induits par le syndrome de la référence glissante (shifting baseline syndrome $e^{4}$, Vera, 2009 p. 28-31) fermée semble disposer d'un statut ambivalent. Pensée comme formation originelle 
réduite à l'état de relique sous la pression des activités humaines, elle serait alors valorisée en tant que référence à une nature intacte mais l'approche dominante de la conservation aurait conduit à privilégier la restauration écologique et l'entretien de paysages plus ouverts dépendants du maintien de pratiques agro-sylvo-pastorales. Il s'agit à présent de comprendre de quelle manière le rewilding se positionne et exploite les modèles de référence contrastés décrits précédemment.

\section{Quelle intégration des modèles de référence dans les discours et pratiques de rewilding en Europe?}

\section{Les références théoriques et temporelles mobilisées dans les stratégies des acteurs du rewilding alimentent un clivage au sein du MNSE}

15 La réintroduction contemporaine du questionnement sur le caractère sauvage de la nature en Europe et les modalités éventuelles de sa préservation puis de sa restauration est marquée par trois étapes bien précises. La première étape se traduit par la recherche des lambeaux de cette nature sauvage au prisme des critères de la wilderness nord-américaine ou tels qu'adaptés pour la conservation à l'échelle internationale par l'Union internationale pour la conservation de la nature (IUCN). La tenue en Écosse en 1983 du troisième congrès mondial de la wilderness organisé par la Wild Foundation puis la création de l'association Wilderness Italia en 1985 (Génot, 2014, p. 107) ou encore les premières expériences de protection des wildlands au Royaume-Uni (Taylor, 2011) constituent des jalons importants de cette première phase. De manière complémentaire à l'analyse proposée ici, la lecture de l'article récent d'Alexandra Locquet et Stéphane Héritier (2020) est essentielle pour saisir la dynamique contemporaine de la construction sociale d'une wilderness européenne.

16 La deuxième étape prend corps aux Pays-Bas avec la mise au point d'une nouvelle doctrine de conservation de la nature, désignée par l'expression Nature Development. Cette nouvelle approche s'inspire et adapte des éléments du rewilding nord-américain. Il s'agit de remplacer l'état de référence historique de paysages agro-sylvo-pastoraux (dans une physionomie proche de celle du milieu du xix ${ }^{e}$ siècle aux Pays-Bas) par un état de référence prénéolithique. Toutefois, contrairement au rewilding CCC, le Nature Development se focalise sur la mobilisation des grands herbivores comme agents de réensauvagement. Le préhistorien Leendert P. Louwe Kooijmans (1995) précise avoir été sollicité par des acteurs de la conservation, dès le milieu des années 1980, pour discuter de la pertinence de l'adoption d'un tel état de référence pour le développement de politiques de conservation aux Pays-Bas. Michiel F. Wallis De Vries (1995), biologiste néerlandais, fait la promotion de cette approche herbivoriste alors que l'expérimentation d'Oostvaardersplassen est engagée depuis quelques années. Dans un article très critique vis-à-vis de cette doctrine, le philosophe de l'environnement Jozef Keulartz (1999) propose une analyse plus précise sur l'émergence du Nature Development. Selon cet auteur, il ne s'agirait plus de protéger et de conserver la nature existante mais d'en produire une nouvelle.

La troisième phase, à partir de la fin des années 1990, est celle du déploiement essentiellement à partir de ce foyer néerlandais - de réformes des stratégies de 
conservation de la nature à l'échelle européenne. Précisément, c'est le caractère sauvage de la nature qui est mis en avant comme alternative à une conservation patrimoniale dépendante de pratiques et de représentations agricoles. C'est lors de cette étape que se structure véritablement le MNSE. Ici encore, le rôle de WWF associé à des opérateurs écotouristiques est décisif. La première initiative mise en place est celle de PAN Parks (Protected Area Network) dont la rhétorique et les objectifs sont très proches de ceux forgés dans les années 1980 (première phase). Il s'agit d'identifier, de mieux protéger et de valoriser les espaces de wilderness en Europe par la mise au point d'un dispositif de labellisation et le développement de l'écotourisme. Le modèle Pan Parks est celui de la constitution de vastes aires protégées dédiées à la libre évolution et, le plus souvent, appuyées sur des périmètres déjà existants (parcs nationaux, réserves). Sur le plan théorique, l'approche défendue est très proche de la version nord-américaine du rewilding CCC. Cependant, une deuxième initiative, Rewilding Europe, va émerger à partir de 2011. Cette ONG a également été soutenue par WWF Pays-Bas. Son approche est résolument centrée sur la mobilisation de l'herbivorie et les idées développées par Frans Vera (Fisher, 2013) qui impliquent souvent la mise en place de clôtures délimitant l'espace de dé-domestication des animaux (ré)introduits afin d'assister leur retour à l'autonomie. De plus, contrairement à Pan Parks qui collabore avec des dispositifs de protection publics, Rewilding Europe mobilise de manière plus nette le levier des financements et des acteurs privés.

Si les deux organisations ${ }^{5}$ se trouvent désormais intégrées à une structure de coordination des initiatives de rewilding en Europe (Wild Europe), elles ont développé leurs propres réseaux de sites labellisés. Elles contestent toutes deux la conservation de la nature trop dépendante du modèle agropastoral mais elles s'opposent sur bien des sujets. Plus spécialement, l'un des points de crispation concerne la mobilisation des états de référence et l'interprétation des paysages forestiers du rewilding. De manière schématique, les tenants du rewilding CCC sont encore très attachés au modèle de la forêt fermée tandis que les herbivoristes fondent souvent leurs projets sur le modèle des boisements pâturés dont la mise en œuvre s'inspirerait et produirait, selon ses détracteurs, des paysages de pseudo-savane. Toutefois, si cette opposition semble nette dans les discours et les positionnements stratégiques à l'échelle européenne (Fisher, 2013), la retrouve-t-on concrètement dans la mise en œuvre d'actions concrètes de rewilding? À cette échelle locale peut-on identifier les mêmes registres de références temporelles, spatiales et paysagères?

\section{Et sur le terrain ? Études de cas}

\section{Le ré-ensauvagement de la vallée de la Côa (au nord-est du Portugal) : un projet herbivoriste?}

19 La vallée de la Côa et le projet de ré-ensauvagement initié sur la réserve privée de Faia Brava constituent un site de référence pour Rewilding Europe. La vallée a d'abord été reconnue pour la richesse de son patrimoine archéologique composé de roches gravées réalisées pour la plupart entre -29000 et $-12000 \mathrm{BP}$. Le site d'art rupestre, classé au patrimoine mondial de l'Unesco (1998), comporte pour l'essentiel des représentations d'animaux (chèvres, bouquetins, aurochs, chevaux, chevreuils, etc.). Un projet de barrage hydroélectrique, puis des projets de carrières, des coupes à blanc ou encore des pratiques de braconnage ont conduit à l'enclenchement d'une mobilisation 
environnementale au début des années 1990 et à un processus de patrimonialisation. Dans un deuxième temps, en 2000, avec l'appui d'une fondation néerlandaise (Stichting Transhumance Natuur, STN), un groupe de biologistes portugais, espagnols, suédois et néerlandais a créé l'association ATNaturaleza ainsi que la réserve naturelle privée de Faia Brava (17 hectares). La réserve, dont l'action ciblait au départ la protection des oiseaux (vautours, aigles de Bonelli), s'étend désormais sur plus de 800 hectares et s'intègre dans d'autres dispositifs de protection (Natura 2000 notamment). Le cœur du projet repose sur la dé-domestication d'herbivores dans un espace clos. Les espèces introduites sont des bovins rustiques et surtout une centaine de poneys de la race ancienne Garrano (espèce native). Dernièrement, le projet a bénéficié du soutien de 2,6 millions d'euros de la part de la fondation Arcadia ${ }^{6}$ via le programme «endangered landscapes" piloté par une structure de l'Uuniversité de Cambridge. Cette aide doit contribuer au changement d'échelle du projet de rewilding dont l'ambition est de créer un corridor de nature sauvage de 120000 hectares calqué sur la vallée de la Côa. La réserve de Faia Brava travaille en synergie avec quatre opérateurs écotouristiques intégrés au réseau de «safaris » constitué par Rewilding Europe.

Ce projet s'inscrit aussi dans la logique d'opportunité de rewilding passif, s'invitant dans les espaces marqués par une forte déprise rurale et un abandon des terres agricoles. Le village le plus proche de la réserve, Cidadelhe, comptait 300 habitants à la fin des années 1950, 150 à la fin des années 1970 et seulement 30 résidents permanents actuellement (DeSilvey et Bartolini, 2019). Le paysage dominant est celui du montado dont la fine mosaïque résulte de pratiques agro-sylvo-pastorales anciennes. Le montado combine des paysages de prairie et des paysages boisés de type pré-bois; ils sont reconnus pour leur valeur culturelle par l'Unesco ${ }^{7}$ et également considérés pour leur haute valeur naturelle (Pinto-Correia et al., 2018). Comme l'illustrent la photo 2 et la vue prospective de Jeroen Helmer (cf. légende de la photo 2), le projet de rewilding tend à naturaliser ce paysage de montado. La physionomie de pré-bois, encore visible malgré la déprise, se trouve mise en scène dans le projet de ré-ensauvagement, brouillant l'origine historique des formes paysagères héritées. L'ancrage de ce projet dans le courant herbivoriste est nuancé par l'évocation du retour des grands prédateurs que l'introduction des herbivores doit favoriser et accompagner. 
Figure 2. Le paysage de la vallée de la Côa, présentant des motifs de montado en déprise

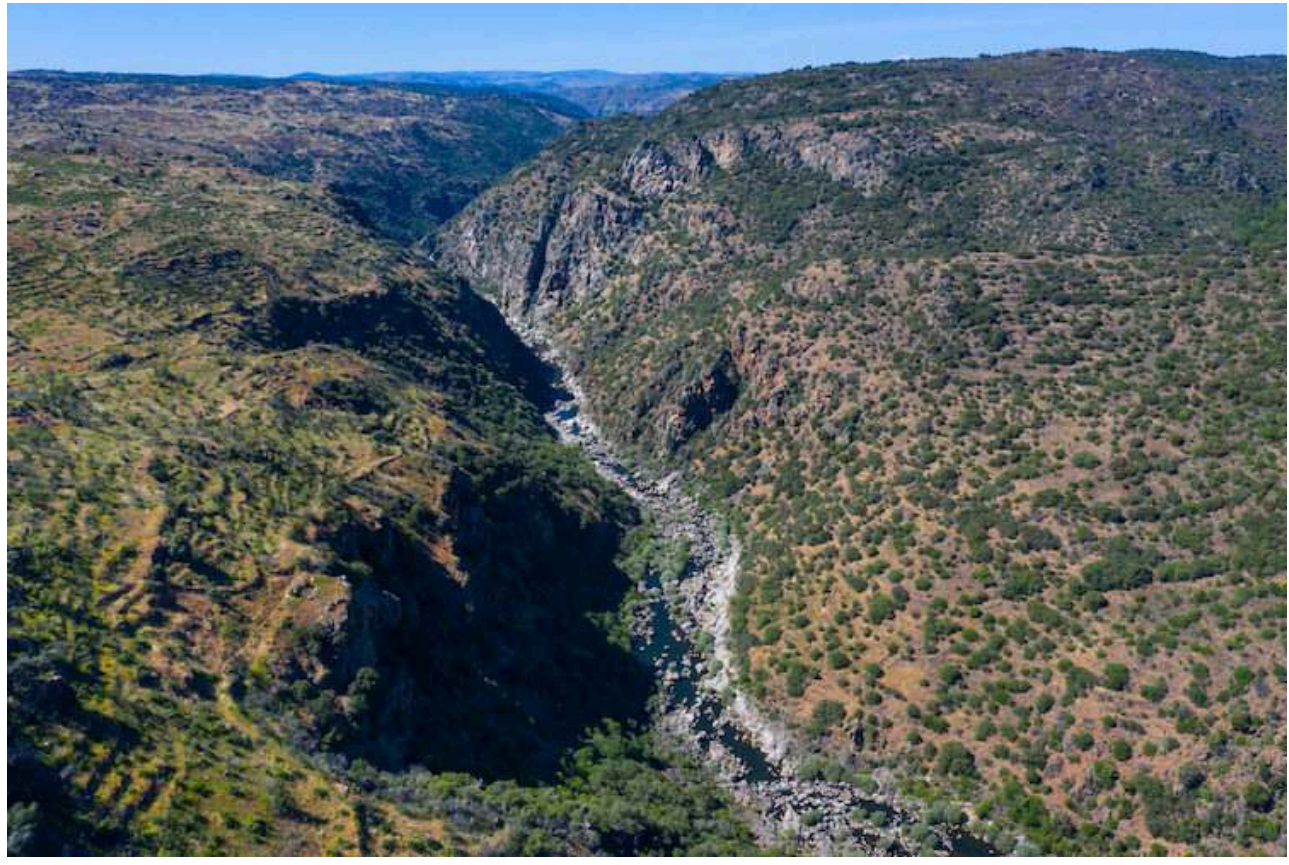

Cette photographie, extraite du site de Rewilding Europe, peut être comparée aux dessins prospectifs réalisés pour le compte de Rewilding Europe par l'artiste Jeroen Helmer tel que celui visible ici : https://rewildingeurope.com/areas/greater-coa-valley/. Les herbivores et les autres animaux produisent un paysage boisé, mais ouvert. Cette vision est à la fois compatible avec l'héritage du montado et avec la vision d'une forêt originelle à la physionomie de savane (cf. thèse de Frans Vera telle que traduite par les acteurs du rewilding).

Source : Juan Carlos Munoz Robredo/Rewilding Europe.

21 Par ailleurs, sur le plan de la mobilisation des temporalités, les porteurs de projets tentent habilement d'exploiter la filiation de la relation entre humains et non-humains en faisant référence aux herbivores figurés sur les roches gravées. Cette relation débute il y a près de 30000 ans (c'est-à-dire bien avant le début de l'Holocène) et se prolongerait par l'action de rewilding. Caitlin DeSilvey et Nadia Bartolini (2019) voient dans cette expérience une opportunité de re-couplage entre humains et non-humains. Ces auteurs considèrent la clôture, qui matérialise l'espace de dé-domestication, comme un dispositif transitionnel ouvrant vers l'autonomie de la nature. Pourtant, dans leur enquête, les auteurs relèvent le témoignage d'un éleveur qui ne comprend pas la nécessité de la clôture et de l'intention de ré-ensauvagement là où l'ensauvagement se réalise sans intervention humaine...

22 Enfin, dernier paradoxe, l'acceptation sociale du projet passe également par une argumentation sur le rôle d'agent de prévention des incendies conféré aux herbivores. Ce projet illustre bien le cheminement tortueux et ambigu du retour et du recours au sauvage. En effet, l'animal dé-domestiqué (ensauvagé) dans un espace clos doit être " apprivoisé » par les habitants locaux et, pour assurer cela, le poney se trouve socialisé par une mission de défense contre le feu!

\section{Ré-ensauvager les Highlands : l'ombre de portée de la mythique forêt calédonienne}

L'Écosse, qui avait accueilli le troisième congrès mondial pour la wilderness, est devenue un des points chauds du MNSE. Les initiatives de protection des wildlands ont été 
initiées par le John Muir Trust (1983), puis par l'écologiste Alan Watson Featherstone qui a fondé l'association Trees For Life en 1986 (Taylor, 2011, p. 20). Enfin, il convient de relever à la même période les premiers pas du projet de restauration des forêts naturelles (native woodlands) le long de la frontière entre l'Écosse et l'Angleterre. Soutenu très tôt par WWF, ce projet est désormais connu sous l'appellation de Borders Forest Trust (BFT, 1996). Ces initiatives sont à replacer dans un contexte de renouvellement de la réflexion sur la couverture forestière du pays. Ce dernier est relativement dépourvu de massifs boisés anciens et fortement concerné par le développement de plantations monospécifiques de résineux non natifs. Ainsi, d'autres organisations tournées vers la reforestation et la foresterie durable apparaissent parallèlement aux projets de conservation à l'exemple de Reforest Scotland (1991). Nous souhaitons, succinctement, discuter ici l'une des initiatives de conservation menée par Trees For Life (TFL) à travers la restauration de la forêt calédonienne dans les hautes terres écossaises. Cette discussion sera conduite en confrontant les analyses géohistoriques, en particulier celles issues des travaux de Christopher Smout (2007 et 2014), et celles plus récentes portant sur les représentations sociales du rewilding des Highlands (Deary, 2015), aux discours des promoteurs de la restauration du caractère sauvage de ces paysages. La forêt calédonienne est aujourd'hui identifiée par les îlots fragmentés de pins sylvestres perçus comme natifs. L'inventaire mené par la commission forestière écossaise en 1998 ajuste le diagnostic : il resterait 84 sites à scots pine couvrant 18000 hectares. Souvent associée à son arbre emblématique, le pin sylvestre (pinus sylvestrus, prenant l'appellation de scots pine), cette forêt a été construite culturellement selon le modèle classique de la couverture continue originelle qui aurait été, dans ce cas précis, dominée par les pins et progressivement dégradée par les activités anthropiques. Christopher Smout (2014) déconstruit cette interprétation culturelle qui semble parfois toujours subsister en toile de fond de certaines initiatives de rewilding. Premièrement, la lecture classique et médiatique tend à assimiler la forêt calédonienne à une forêt composée essentiellement de pins. Smout récuse cela en dissociant, dans la reconstitution de l'évolution de la couverture forestière, les pins des autres espèces. Ce faisant, il distingue les paysages forestiers boréaux des Highlands, comprenant des pins dans une mosaïque lâche, associés à des bruyères, myrtilliers et genévriers, et ceux des plaines dans lesquels les pins étaient rares et les feuillus dominaient. Deuxièmement, l'auteur démontre l'inexactitude de l'idée selon laquelle les forêts de pins auraient été essentiellement détruites par les activités humaines durant la période antique. Ses recherches et celles des archéologues et des spécialistes des paléo-environnements ont permis d'attester que la dégradation de la forêt, composée de pins et de feuillus (couverture maximum, -600 BP), a eu lieu avant la mise en route d'activités agricoles significatives pour des raisons climatiques. Une phase humide et fraîche (-4000 BP) dans les Highlands a progressivement produit des paysages de landes humides et de tourbières. À partir de la fin du XVIII ${ }^{\mathrm{e}}$ siècle, les îlots de scots pines auraient été réduits et contraints par l'introduction de l'élevage ovin et le développement du chevreuil pour la pratique élitaire de la chasse. Ces mutations sont particulièrement sensibles dans l'histoire des Highlands écossaises car elles entrent en résonance avec le mouvement des Clearances engageant le déguerpissement des habitants par les grands propriétaires terriens anglais (Deary, 2015, p. 97 et 101). Christopher Smout comme Ian D. Rotherham (2014) mettent en garde les rewilders contre la tentation de vouloir rétablir l'arbre et la forêt partout, y compris là où ils n'ont jamais été présents de manière durable. 
Dès 1986, le projet porté par TFL intègre et dépasse la question du sauvetage patrimonial du scot pines et de la forêt calédonienne. Il s'agit bien, dès le départ, d'un projet de ré-ensauvagement à large échelle, très proche de la conception nordaméricaine du rewilding CCC. En effet, le projet repose sur l'identification, dans les Highlands, d'un espace de plus de 238000 hectares qui serait favorable à la restauration d'une forêt naturelle en raison de son isolement, de la faible densité de population humaine et de son faible niveau d'aménagement et d'exploitation. La restauration de cette forêt naturelle de grande dimension, strictement protégée des activités extractives, serait couplée aux réintroductions du castor, du sanglier et de prédateurs clés tels que le loup et le lynx (Watson Featherstone, 1997, p. 3). Sur le plan de la reconstitution de la forêt, TFL met essentiellement en avant la régénération naturelle. Il s'agit de commencer par prévenir les dégradations des îlots de scot pines en les protégeant des chevreuils et des moutons (protection par des clôtures temporaires). La stratégie comprend dans un deuxième temps des actions de replantation d'essences locales de pins et de feuillus. Cette approche démontre une certaine évolution dans la conception de la forêt calédonienne en évitant l'assimilation à la forêt de pins dénoncée par Smout (2014). Cependant, les replantations font débat au sein même des mouvements environnementalistes (authenticité des boisements, principe étendu de la non-intervention). Le plan de ré-ensauvagement devait également comprendre un volet de dés-aménagement des artéfacts (routes, barrages, conduites, etc.).

Le leader de TFL (Watson Featherstone) est parfaitement conscient des obstacles sociaux et politiques à la mise en œuvre d'un tel projet mais il défend fermement l'intégralité de sa vision de restauration de la wilderness ${ }^{8}$ à large échelle. Cette " vision " est parfaitement concordante avec celle reprise par Pan Parks puis par la European Wilderness Society (cf. note infra 4). TFL se présente elle-même comme une association caritative ayant pour objectif le rewilding ${ }^{9}$ des Highlands écossaises. La structure ne s'est pas engagée dans la maîtrise du foncier, elle coopère et contractualise avec des opérateurs variés, y compris avec la compagnie de foresterie nationale. Sur le plan des temporalités, TFL demeure globalement fidèle aux interprétations historiques actualisées (Smout, 2014). En particulier, les éléments de discours portant sur les conditions d'émergence, de colonisation et de retrait des pins sont conformes à la chronologie présentée ci-avant. Toutefois, l'interprétation des causes et du rythme du retrait est partiellement en adéquation avec la lecture historienne. Si le récit de la déforestation présenté par l'association ${ }^{10}$ reconnaît bien une phase de retrait précoce, il y a 4500 ans à la faveur d'une période humide et froide, et la réduction par deux de la couverture maximale au moment de l'arrivée des Romains ( $\mathrm{I}^{\mathrm{er}}$ siècle), le récit de TFL insiste davantage sur les facteurs de dégradation anthropiques. Les activités humaines, selon l'association, auraient pu jouer un rôle décisif dans la régression de la forêt dès l'arrivée des premiers agriculteurs, c'est-à-dire il y a environ 3900 ans. Cette vision d'une dégradation anthropique précoce et significative est aussi partagée par d'autres scientifiques (Brown et al., 2012). En revanche, TFL évoque plus précisément les effets délétères des replantations industrielles à partir des années 1920. Enfin, le projet de réensauvagement repose sur une vision futuriste de long terme (250 ans).

Aujourd'hui, l'action de TFL se trouve paradoxalement référencée dans le réseau de Rewilding Europe et de sa déclinaison nationale, Rewilding Britain. Comme nous l'avons vu précédemment, ces structures sont connues pour leur approche herbivoriste et elles sont parfois même vivement critiquées pour les actions qui découlent de cet 
ancrage (Fisher, 2013). Au cours des cinq dernières années, le ré-ensauvagement des Highlands a motivé de nouvelles initiatives. Celles-ci sont caractérisées par deux dimensions fondamentales. Premièrement, on assiste à une volonté de coordination des actions de rewilding à large échelle dans le Nord de l'Écosse. Dans cette perspective, le rewilding prend appui sur les périmètres et les dispositifs institutionnels du Parc national de Cairngorms créé en 2003 et de quatre réserves naturelles. La coordination des différentes initiatives se concrétise à travers le projet Cairngorms Connect qui s'étend sur $600 \mathrm{~km}^{2}$ et comprend 9800 hectares de boisements, dont la moitié est composée de scot pines. La deuxième dimension du rewilding en Écosse est de nature financière. En effet, ce projet de grande ampleur a bénéficié - comme celui de la vallée de la Côa d'investissements privés très importants (23 millions de livres) via le programme Endangered Landspaces soutenu par la fondation Arcadia ${ }^{11}$. Ce soutien révèle également l'implication d'autres richissimes acteurs comme le danois Anders Holch Povlsen. Cependant, la multiplication des projets, malgré la volonté de coordination, édulcore et brouille sensiblement les principes défendus par les pionniers de TFL. Le rewilding des Highlands tend à devenir une vaste entreprise de requalification territoriale et de marketing. Il donne lieu à un puissant processus d'esthétisation mobilisant l'arbre et la forêt dans un espace dont les paysages très ouverts sont considérés comme sauvages dans les représentations populaires (Deary, 2015, photos 3 et 4).

Figure 3. La forêt des Highlands, réserve naturelle nationale de Glen Affric

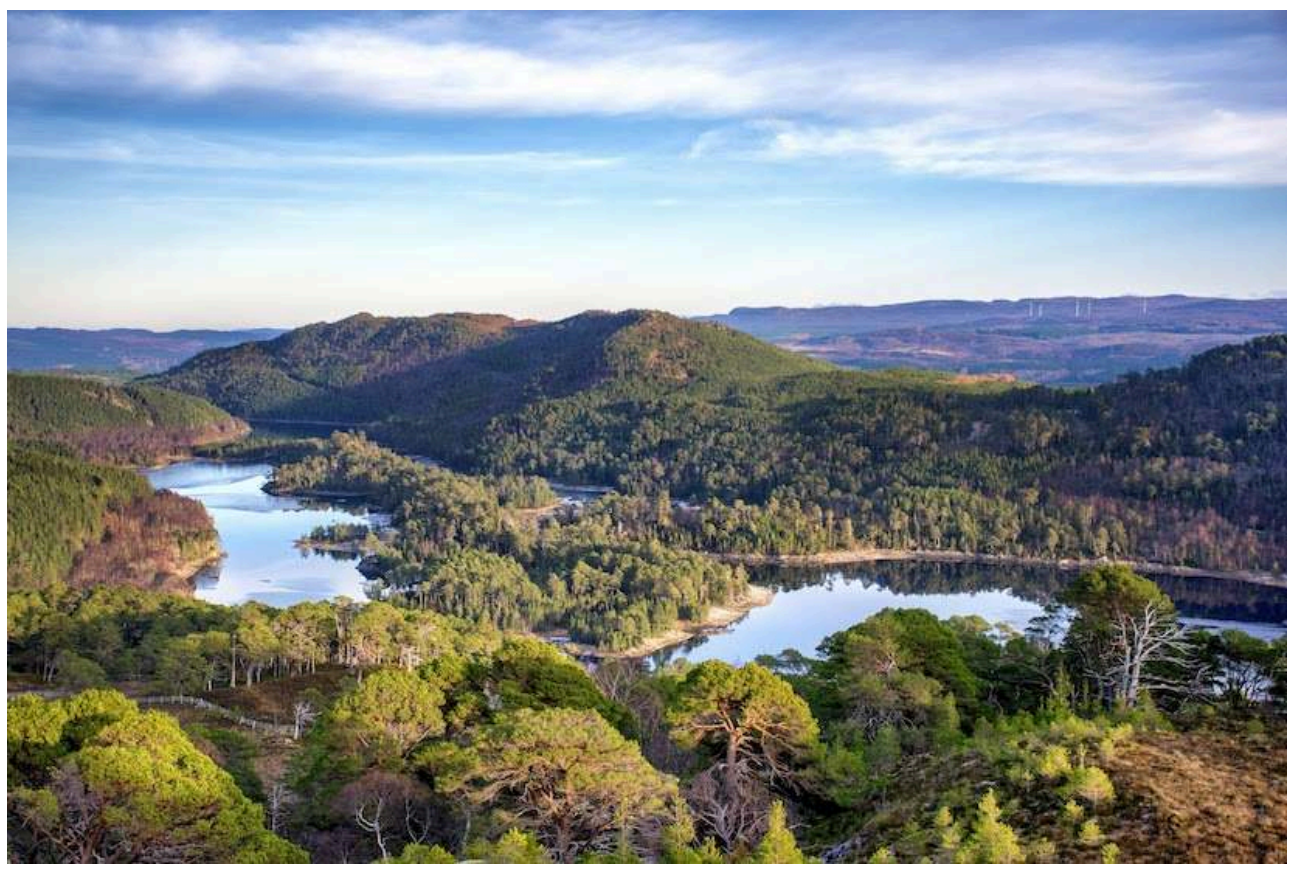

Une vue esthétisée de la forêt calédonienne associant les pins et les feuillus.

Source : Chris Charles Photography@chrisscharless - Trees for Life. 
Figure 4. Les hautes terres écossaises : paysages du surpâturage ou paysage boisé ré-ensauvagé ?

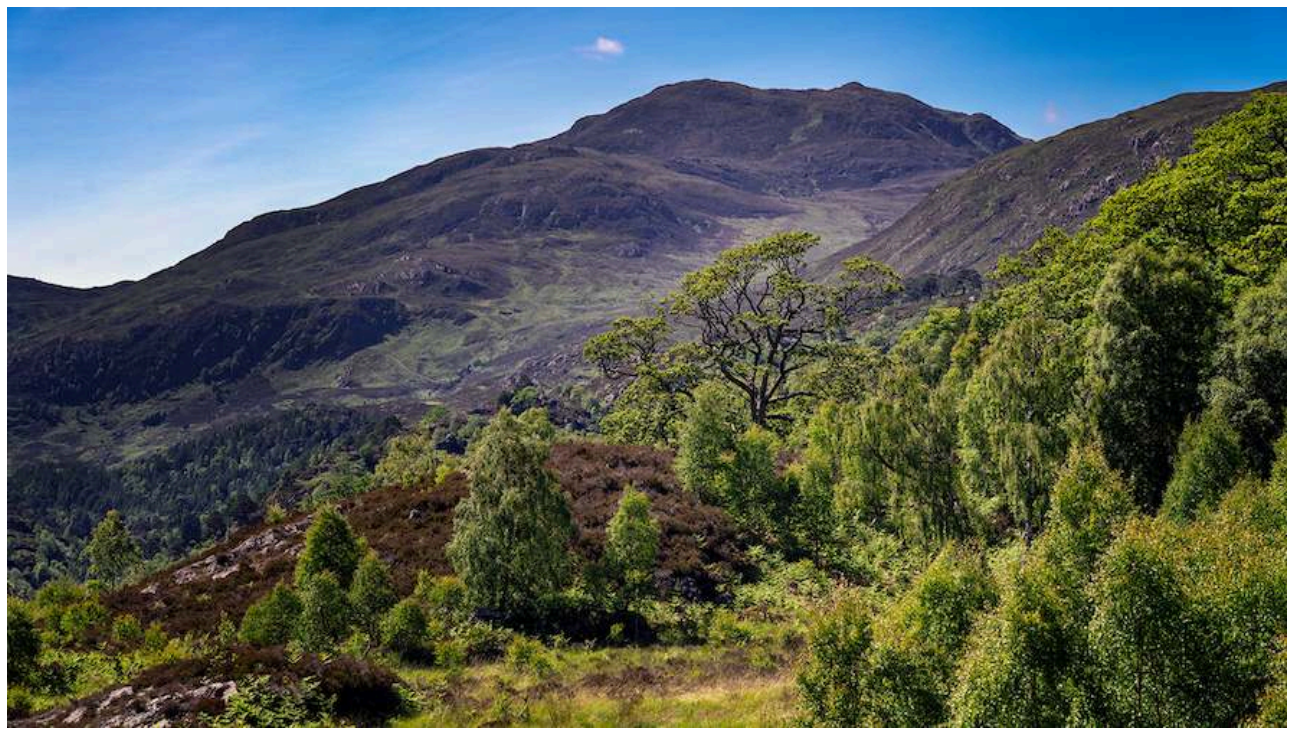

Cette prise de vue est également extraite du site Web de Trees of Life. En arrière-plan, les uplands dénudés, souvent mobilisés pour évoquer les paysages sauvages écossais (et du Royaume-Uni en général). Ces paysages sont aussi considérés par les rewilders comme des déserts écologiques ou des « sheepwrecked landscapes », symboles de la dégradation anthropique (surpâturage).

Source : Chris Charles Photography@chrisscharless - Trees for Life.

\section{Conclusion}

La déprise agricole aurait - pour reprendre l'analyse de Pascal Marty - enclenché à l'échelle européenne, depuis la fin de la Seconde Guerre mondiale, un processus de transition conduisant la mosaïque agro-sylvo-pastorale vers « une autre structure paysagère qui, culturellement, est plutôt assimilée à la nature sauvage » (Marty, 2012, p. 169). Cet ensauvagement se traduirait par une dynamique de succession classique conduisant à la forêt. Le retour de la forêt sur les terres marginales progressivement abandonnées constituerait l'autre face des mutations paysagères (remembrement) induites par l'industrialisation des moyens de production et la concentration affectant le secteur agricole. Cet ensauvagement, parfois vécu comme une perte de maîtrise du territoire et une dégradation paysagère, était déjà redouté dès la fin des années 1980 . Aujourd'hui, un tel dynamisme végétal non orienté vers un projet d'exploitation économique, de mise en ordre du territoire et de jardinage des paysages est au contraire très favorablement accueilli par certains écologues et militants. Ainsi, Annik Schnitzler et Jean-Claude Génot (2012) appellent à la reconnaissance de la valeur de cette nature en évolution libre, non instrumentalisée, voire "décolonisée ». Ce retour du ligneux est un processus récent mais suffisamment puissant pour être perçu par les populations locales, les visiteurs, les scientifiques comme les profanes. Il est souhaité ou rejeté, mais il ne laisse pas indifférent surtout lorsque le boisement spontané précède ou accompagne le retour d'autres agents non humains de l'ensauvagement (des sangliers aux loups).

Les caractéristiques de cette transition vers des paysages beaucoup plus boisés orientent le rewilding en Europe vers des temporalités a priori contrastées mais parfois combinées. La première conduirait à rechercher et à protéger, voire à recréer, les forêts 
les plus anciennes ou leurs reliques. La seconde traduirait un basculement de la flèche du temps. Il ne serait plus question de fixer dans le passé un état de référence. Au contraire, l'avènement des paysages de la féralité s'inscrirait sur des trajectoires «non pilotées " par l'action humaine. Les processus naturels, sans intervention gestionnaire, configureraient une nature changeante et incertaine censée proposer une rupture expérientielle. En effet, à la scénographie des paysages agro-sylvo-pastoraux patrimonialisés, la féralité et l'ensauvagement substitueraient une esthétique de la libre évolution de la nature. De manière complémentaire, les différentes conceptions du rewilding semblent aboutir - sur le plan des postures et des discours stratégiques - à des natures et des paysages très différents. L'imaginaire d'une forêt continue, médiatisé par l'esthétique de quelques emblèmes (Bialowieza et les forêts de l'Est par exemple), est sous-tendu par la conception du rewilding CCC. À l'inverse, la conception herbivoriste du rewilding privilégierait la forêt à l'apparence de savane en tant qu'état de référence et horizon d'attente. Les représentations paysagères véhiculées relèvent ici sans doute d'une vision alternative de la forêt primaire européenne. Ces représentations dénotent aussi l'influence d'autres références plus exotiques et coloniales (Serengeti, safaris).

Cependant, les études de cas nous incitent à nuancer les effets d'oppositions liées à cette matrice de conceptions et de discours divergents. En effet, les exemples mettent au jour la complexité, les inflexions et les paradoxes qui caractérisent les références spatiales et temporelles dans la mise en œuvre concrète des projets de rewilding. Les effets d'intégration à des réseaux européens, puis nationaux, se mêlent aux enjeux locaux pour alimenter des récits mouvants du rapport au sauvage et du rapport aux lieux. Ces mises en récit encapsulent des imaginaires et des savoirs savants. Les histoires médiatisées par les textes, images et vidéos participent d'un processus d'esthétisation (voire d'artialisation) qui arrime la nature forestière à des physionomies paysagères. On passe ainsi littéralement de la conception normative scientifique à de véritables « visions ». Celles-là sont censées susciter l'émotion et renforcer l'adhésion à la stratégie de ré-ensauvagement. S'il existe de très bonnes raisons à vouloir réinsuffler de l'émotion dans les pratiques de ménagement de l'espace, de conservation de la nature ou d'élaboration de paysage (Le Lay, 2019), il conviendrait cependant d'être prudent et de veiller, dans le cas du ré-ensauvagement des paysages forestiers, à ce que le flux d'images diffusées ne soit pas vécu comme un «forçage » des sensibilités locales qui s'expriment dans le rapport aux lieux habités.

\section{BIBLIOGRAPHIE}

Agnoletti, M. et Emanueli, F., (eds), Biocultural diversity in Europe, Florence, Springer, 2016.

Barraud, R., Andreu-Boussut, V., Chadenas, C., Portal, C. et Guyot, S., « Ensauvagement et réensauvagement de l'Europe : controverse et postures scientifiques ", Bulletin de l'Association des géographes français, vol. 96, $\mathrm{n}^{\circ}$ 2, 2019, p. 301-318. 
Barraud, R., Périgord, M., « L'Europe ensauvagée : émergence d'une nouvelle forme de patrimonialisation de la nature ? ", L'Espace Géographique, t. 42, nº 3, 2013, p. 254-269, URL : https://www.cairn.info/revue-espace-geographique-2013-3-page-254.htm.

Barthod, C., « Le retour du débat sur la wilderness ", Revue forestière française, $\mathrm{n}^{\circ}$ 1, 2010, p. 57-70. Brown, C., McMorran, R. et Price, M. F., « Rewilding - A new paradigm for nature conservation in Scotland », Scottish Geographical Journal, vol. 127, nº 4, 2012, p. 288-314.

Carbonnelle, S. et Lezaca-Rojas, S., « Bialowieza, la réaction trop tardive des institutions », $L a$ Lettre de forêts sauvages, $\mathrm{n}^{\circ} 18,2017$, p. 4-6.

Claval, P., « Passer à l'avenir. Le rôle des normes et des horizons d'attentes », Cahiers de géographie du Québec, vol. 51, nº 144, 2007, p. 347-367.

Deary, H., « Restoring wildness to the Scottish Highlands », dans Hourdequin, M., Havlick, D. G. (eds.), Restoring layerd landscapes: History, Ecology, and Culture, Oxford, Oxford University Press, 2015.

Debarbieux, B., « Imaginaire géographique », dans Lévy, J. Lussault, M., (dir.), Dictionnaire de la géographie et de l'espace des sociétés, Paris, Belin, 2005.

DeSilvey, C. et Bartolini, N., « Where horses run free? Autonomy, temporality and rewilding in the Côa Valley, Portugal ", Transactions of the Institute of British Geographers, vol. 44, n 1, 2019, p. 94-109.

Fisher, M., « Wild nature reclaiming man-made landscapes », Ecos, vol. 34, n 2, 2013, p. 50-58

Franklin, S., « Bialowieza Forest, Poland: represnetations, myth, and the politics of dispossession », Environment and Planning A, vol. 34, 2002, p. 1459-1485.

Génot, J.-C., « Naturalité et biologie de la conservation », dans Gauthier-Clerc M., Mesléard F. et Blondel J., (dir.), Sciences de la conservation, Louvain-la-Neuve, De Boeck, 2014.

Génot, J-C., Instinct Nature, Paris, Sang de la Terre, 2010.

Harrison, R., Forêt. Essai sur l'imaginaire occidental, Paris, Flammarion, 1992.

Hartel, T. et Plieninger, T., (eds.), European wood-pastures in transition. A social-ecological approach, London/New York, Earthscan/Routledge, 2014.

Hedemann, O., L'histoire de la forêt de Bialowieza (jusqu'en 1798), Varsovie, Institut de recherche des forêts domaniales de Pologne, Série A, nº 41, 1939.

Jepson, P., « A Rewilding Agenda for Europe: Creating a Network of Experimental Reserves », Ecography, n 39, 2016, p. 117-124.

Jørgensen, D., « Competing ideas of "natural” in a dam removal controversy », Water Alternatives, vol. 10, $\mathrm{n}^{\circ}$ 3, 2017, p. 840-852.

Jørgensen, D., « Rethinking Rewilding », Geoforum, vol. 65, 2015, p. 482-488.

Keulartz, J., « Engineering the environment: the politics of nature development ", dans Fisher, F. et Hajer, M. A. (eds.), Living with nature, Oxford University Press, 1999.

Kirby, K. J., « What might a British forest-landscape driven by large herbivores look like? », Peterborough, English Nature (Natural England), Research report, $n^{\circ}$ 530-1, 2003.

Larrère, R., « La notion de climax : modèle d'une nature sauvage ", Études Rurales, n 129-130, 1993, p. 15-31, URL : https://www.persee.fr/doc/rural_0014-2182_1993_num_129_1_3399. 
Latour, B., Politiques de la nature. Comment faire entrer les sciences en démocratie, Paris, La Découverte, 1999.

Le Lay, Y-F., « Emotionscapes. S’é-mou-voir des situations géographiques », HDR, ENS Lyon, 2019.

Lepart, J., Marty, P., « Les conceptions normatives du paysage : Le cas des Grands Causses », Natures Sciences Sociétés, 2000, vol. 8, n 4, p. 16-25.

Locquet, A., Héritier, S., « Interrogation autour de la nature et du sauvage à propos de l'établissement de wilderness areas en Europe ", Cybergeo. Revue européenne de géographie, 2020, article $\mathrm{n}^{\circ}$ 946, URL : http://journals.openedition.org/cybergeo/34986 ; DOI : https://doi.org/ 10.4000/cybergeo.34986

Lorimer, J., Sandom, C., Jepson, P., Doughty, C. E., Barua, M. et kirby, k., « Rewilding: Science, Practice, and Politics ", Annual Review of Environment and Resources, vol. 40, 2015, p. 39-62.

Louwe Kooijmans, L. P., « Prehistory or Paradise? Prehistory as a reference for Modern Nature Development, the Dutch case », Mededelmgen Rifts Geologische Dienst, n52, 1995, p. 415-424.

Marty, P., « Fin du paysage agraire et retour de la nature sauvage ? ", dans Bergé, A., Collot, M. et Mottet, J. (Eds.), Paysages européens et mondialisation, Seyssel, Champ Vallon, 2012.

Ollitrault, S., « De la caméra à la pétition-web : le répertoire médiatique des écologistes ", Réseaux, vol. 98,1999 , p. 153-185.

Peterken, G. F., Woodland conservation and management, London / New York, Chapman and Hall, 1981.

Pezzullo, P. C., Cox, R., (eds.), Environmental communication and the public sphere, Los Angeles, SAGE publishing, 2018.

Pinto-Correia, T., Guiomar, N., Ferraz-de-Oliveira, M. I. et al., « Progress in identifying High Nature Value Montados: impacts of grazing on hardwood rangeland biodiversity », Rangeland Ecology and Management, vol. 71, $\mathrm{n}^{\circ}$ 5, 2018, p. 612-625.

Ribaut, J.-P., «L'action du Conseil de l'Europe en matière de zones protégées », Revue forestière française, vol. XLIII, nº spécial « patrimoine », 1991, p. 213-220.

Rochel, X., «La fin des vides en forêt : essais de cartographie historique », Revue forestière française, vol. LXVII, $\mathrm{n}^{\circ}$ 6, 2015, p. 539-556.

Rotherham, I., Eco-history. An introduction to biodiversity and conservation, Cambridge, The White Horse Press, 2014.

Rotherham, I. (eds.), Cultural severance and the Environment, Dordrech, Springer, 2013.

Sabatini, F. M., Burrascano, S., Keeton, W. S. et al., " Where are Europe's last primary forests? », Diversity and Distributions, vol. 24, $\mathrm{n}^{\circ} 10,2017$, p. 1-14.

Schama, S., Le Paysage et la Mémoire (1995), Paris, Le Seuil, 1999.

Schnitzler, A., Génot, J.-C., La France des friches. De la ruralité à la féralité, Versailles, Éditions Quae, 2012.

Smout, C., " The history and the myth of Scots Pine », Scottish Forestry, $\mathrm{n}^{\circ}$ 68, 2014, 7 p.

Smout, C., Mac Donald, A. R., Watson, F., A history of the Native Woodlands of Scotland (1500-1920), Edinburgh University Press, 2007.

Soulé, M., Noss, R., « Rewilding and Biodiversity: Complementary Goals for Continental Conservation », Wild Earth, vol. 8, n 3, 1998, p. 18-28. 
Taylor, P., (dir.), Rewilding, ECOS writing on wildland and conservation values, Ethos-UK, 2011.

Tsing, A., Le Champignon de la fin du monde. Sur les possibilités de vivre dans les ruines du capitalisme, Paris, La Découverte, 2017.

Vallauri, D., « Biodiversité, naturalité, humanité », Marseille, MEDD/WWF, 2007.

Vera, F., « Large-Scale nature development - the Oostvaardersplassen », British Wildlife, juin, 2009, p. 28-36.

Vera, F., Grazing ecology and forest history, Wallingford, Caby Publishing, 2000.

Wallis De Vries, M. F., « Large herbivores and the design of large-scale nature reserve in Western Europe ", Conservation Biology, vol. 9, n 1, 1995, p. 25-33.

Watson Featherstone, A., « The Wild Heart of the Highlands ", ECOS, vol. 18, n² 2, 1997, p. 48-61.

\section{NOTES}

1. Comme l'illustre l'appel récent lancé par Francis Hallé (2019) dédié à la reconstitution d'une forêt primaire en Europe : https://www.lemonde.fr/idees/article/2019/10/07/il-y-a-urgence-areconstruire-de-grandes-forets-primaires_6014470_3232.html

2. Caractérise l'ensauvagement d'une espèce qui a été domestiquée.

3. Frans Vera est un biologiste néerlandais, investi dans le champ de la conservation de la nature. Depuis la fin des années 1990, il mène des recherches sur le rôle des grands herbivores dans la composition et la dynamique forestière de l'Europe tempérée de plaine.

4. Cette idée a été formalisée par le spécialiste des milieux marins français Daniel Pauly en 1995 : le glissement imperceptible de l'état de référence d'une génération à l'autre masque l'ampleur de la crise écologique engagée sur le long terme.

5. L'ONG Pan Parks a été officiellement liquidée en 2013 mais son projet a été repris dans une nouvelle structure, la European Wilderness Society, fondée en 2014 et soutenue par l'ONG « mère » internationale, la Wild Foundation.

6. Fondation dirigée et financée par Lisbet $K$. Rausing et Peter Baldwin. Le grand-père de Rausing est le fondateur de la compagnie suédoise Tetra Pak et la famille Rausing est propriétaire de la compagnie Ingleby - Farms and Forests.

7. Cf https://whc.unesco.org/en/tentativelists/6210/

8. Alan Watson Featherstone utilise aussi l'expression self-willed-land en référence aux travaux du forestier américain Aldo Leopold. Cette expression est régulièrement associée par l'auteur à l'expression : «nature knows best ».

9. C'est le terme utilisé par TFL, par exemple ici : https://treesforlife.org.uk/about-us/

10. Ce récit est publié sur le site de TFL : https://treesforlife.org.uk/into-the-forest/habitats-andecology/human-impacts/deforestation/

11. Cf. article du Guardian publié le 7/10/2018: https://www.theguardian.com/uk-news/2018/ oct/07/caledonias-lost-forest-restored-to-glory-in-rewilding-cairngorms 


\section{RÉSUMÉS}

Les acteurs du mouvement de promotion de la nature sauvage et du ré-ensauvagement mobilisent des références spatiales et temporelles très distinctes afin de fonder leurs stratégies et projets. Plus particulièrement, les différentes formes de rewilding révèlent des imaginaires et des conceptions scientifiques (normatives) des paysages forestiers contrastés. Ainsi, le modèle d'une forêt primaire européenne se développant au début de l'Holocène en tant que couverture dense et continue est concurrencé depuis le début des années 2000 par d'autres visions, dont celle proposée par Frans Vera. Ce dernier défend l'idée d'une forêt postglaciaire à " physionomie de savane " fortement influencée dans sa dynamique et sa structure par les grands herbivores. Par ailleurs, si les acteurs du rewilding « regardent » vers le passé, ils élaborent également des projets futuristes, conçus sur le temps long. La mise en œuvre d'opérations de ré-ensauvagement intègre des traductions simplifiées de ces modèles et aussi la complexité des histoires locales. Cela contribue à une mise en récit diversifiée de rapports renouvelés à la nature sauvage en Europe.

Actors in the movement for the promotion of nature and rewilding rely on very distinct spatial and temporal references upon which to ground their strategies and projects. The different forms of rewilding reveal imaginary representations and scientific (normative) conceptions of contrasting forest landscapes. Since the early 2000s, the model of a European primary forest developed at the beginning of the Holocene as a dense continuous cover has competed with other hypotheses, including the one put forward by Frans Vera. The latter defends the idea of a postglacial forest with a "savannah-like aspect" the dynamics and structure of which were strongly influenced by large herbivorous animals. Moreover, while the proponents of rewilding "look" to the past, they also develop futuristic projects designed over long periods of time. The implementation of rewilding operations integrates simple transpositions of these models as well as the complexity of local histories. This contributes to a diversified narrative of renewed relationships to wilderness in Europe.

\section{INDEX}

Mots-clés : rewilding, forêt, temporalité, discours, projets, Europe

Keywords : rewilding, forest, temporality, speech, projects, Europe

\section{AUTEUR}

\section{RÉGIS BARRAUD}

Régis Barraud est géographe, maître de conférences à l'université de Poitiers, Ruralités EA 2252. Ses recherches s'inscrivent dans le champ de la géographie sociale de l'environnement. regis.barraud[at]univ-poitiers[dot]fr https://ruralites.labo.univ-poitiers.fr/membres/regis-barraud/ 